\title{
Biometrics Fingerprint Recognition using Discrete Cosine Transform (DCT)
}

\author{
Muzhir Shaban Al-ANI \\ AL- Anbar University \\ Ramadi- Anbar- Iraq
}

\author{
Wasan M. Al-Aloosi \\ AL- Anbar University \\ Ramadi- Anbar- Iraq
}

\begin{abstract}
Biometric systems based on the fingerprint recognition are considered one of the most important identification techniques. It is a successful way to determine the identity of the person that cannot be faked or stolen easily. This study aims to identify fingerprint images through several steps and extract the features based on DCT technique. The fingerprint image is divided into sub-blocks and allows evaluating the statistical features from the DCT Coefficients .The matching process is implemented using the correlation between fingerprint images. The obtained results include an efficient recognition using DCT.These programs are implemented via MATLAB environment.
\end{abstract}

\section{Keywords}

Biometrics, Fingerprint, Recognition, Feature Extraction, DCT.

\section{INTRODUCTION}

Recently, we see increasing the demand of strict security systems to protect places and people and information from any unwanted interventions of unauthorized. Raced scientists for innovation methods and exploitation techniques are employed to serve the security requirements that are increasingly important day by day, especially in light of the changes of international, especially after what has become known as 11 of September that changed the reality point of view and non-standards and increased the necessities of tightening control and prevent any security breaches.

Biometrics approaches are technologies used for measuring and analyzing a person's unique characteristics. There are two types of biometrics: behavioral and physical. Behavioral biometrics systems are generally used for verification while physical biometrics can be used for either identification or verification [1].These characteristics are unique to individuals hence can be used to verify or identify a person [2].The need to facilitate the increasing levels of international trade, migration and travel while combating organized crime and national security threats, has placed identity management high on the agenda of governments world-wide. Biometrics combines two processes key to verifying the identity of an individual [3]. A typical biometric system is comprised of five integrated components [4]:A sensor, Signal processing algorithms, data storage, a matching algorithm and a decision process .There are two modes for biometric systems: Verification it is necessary for the person to claim an identity through an identification number, user name, or other means. The system gathers the input data and compares it to the template data previously stored for that person. This comparison is a one-to-one comparison, and the system is only trying to verify that the person attempting to gain access is truly who he claims to be. If the input data does not match the template data, the system will deny access [6]. The second operating mode is called identification. The system compares the input data to all sets of template data already stored. This operation is a one-to-many comparison, and there is no need for the person to claim an initial identity There are two types of biometric system [4].The most prominent types of measures to date are:

- Biometric Systems Based on Physiological Characteristics. They include biometrics which is derived from a direct measurement of a part of a human body.

1. Fingerprints and palm prints: - No two persons have exactly the same arrangement of ridge patterns, Personal characteristics may change, but fingerprints do not. Each finger print is as close to being 'unique' as you can get. Fingerprints therefore remain the most powerful and widely used biometric technology in forensics.

2. Iris: The iris is the annular region of the eye bounded by the pupil and the sclera (white of the eye) on either side. Visual texture of the human iris is posited to be distinctive for each person and each eye. These systems are attractive because the pattern variability of the iris among different persons is extremely large.[6].

3. Face: - is one of the most acceptable biometric systems, and it is one of the most common methods of recognition that humans use in their daily visual interactions. The method of acquiring face an image is nonintrusive [4]. Current researches are concerned with involving development of more robust approaches that account for changes in lighting, expression, and aging, where potential variations exist for each given person [16]. Also, other problem areas have been investigated; they include dealing with glasses, facial hair, and makeup [6].

4.Hand geometry: - the capture of measurements encompassing the width, height and length of the fingers, distances between joints and shapes of the knuckles. While reasonably diverse, the geometry of an individual's hands is not necessarily unique.

- Biometric Systems Based on Behavioral Characteristics:

Observes the change in some attributes over a short period of time. This type of systems extracts characteristics based on an action performed by an individual; they are based on indirect measures of the characteristic of the human form. The most prominent and successful types are:

1. Signature recognition: - analyses a series of movements that contain unique biometric data such as personal rhythm acceleration and pressure flow. Since these movements can vary with each signing, differentiating between the consistent and the behavioral parts of a signature is difficult.

2. Voice recognition: - focuses on differences resulting from the shape of vocal tracts and learned speaking habits. Operates best when there's no background noise.

3. Gait recognition: - captures a sequence of images for analysis of how an individual walks. Still in an early stage of 
research \& development. Studies have shown that gait changes over time and is also affected by clothes, footwear, walking surfaces, and other conditions. This system can be implemented covertly for enhancing security. The future aspects in biometrics are body odor identification and the human ear canal for identification.

\section{FINGERPRINT RECOGNITION}

Fingerprint Identification is the method of identification using the impressions made by the minute ridge formations or patterns found on the fingertips.

\subsection{Background}

No two persons have exactly the same arrangement of ridge patterns, and the patterns of any one individual remain unchanged throughout life. Fingerprint recognition refers to the automated method of verifying a match between two human fingerprints. Fingerprints are one of many forms of biometrics used to identify an individual and verify the identity [15]. It is one of the most mature biometric technologies and is suitable for a large number of recognition applications [17]. A fingerprint is made of a series of ridges and furrows on the surface of the finger. The uniqueness of a fingerprint can be determined by the pattern of ridges and furrows as well as the minutiae points [13].Fingerprint recognition is one of the popular biometric techniques. It is mainly used in the identification of a person and in criminal investigations. The first detailed description of the anatomical formation of fingerprints was made by Mayer in 1788 . Purkinje, in 1823, proposed the first fingerprint classification scheme, which classified fingerprints into nine categories according to the ridge configurations, Sir Francis Galton (1888) conducted an extensive study on fingerprints. He introduced the minutiae features for comparing fingerprints. The biological principles of fingerprints (Moenssens, 1971) are summarized below [5]:

1. Individual epidermal ridges and furrows have different characteristics for different fingerprints.

2 . The configuration types are individually variable, but they vary within limits that allow for a systematic classification.

3. The configurations and minute details are permanent.

\section{Advantages}

- $\quad$ Easy to use, with some training

- Some systems require little space

- Has proven effective in many large scale systems over years of use.

- Fingerprints are unique to each finger of each individual

- And the ridge arrangement remains permanent during one's lifetime

\section{Disadvantages}

- Privacy concerns of criminal implications

- Health or societal concerns with touching a sensor used by countless individuals

- Collection of high quality nail-to-nail images requires training and skill, but current flat reader technology is very robust

- An individual's age and occupation may cause some sensors difficulty in capturing a complete and accurate
Uses the reader to take a fingerprint of the person to be proof of his identity as embodied his job at emulate the work of the human analyst, it is founded two tasks: a picture of a fingerprint and Verification of fingerprint taken with a footprint that is stored and by comparing the samples in the database.

\subsection{Literature Survey}

Several researchers in the field of fingerprint recognition developed different recognition methods. Some of these researches are summarized below:

- Abdullah (2008) [7]. The method provides good results especially with dry/neutral type, with the oily images the results are not as good. Implementation of the enhancement algorithm requires three steps: determination of ridge directions, formation of the filtering mask and its application on the image and finally the binarization operations.

- Marasco (2012) [8]. The proposed investigation focuses on combining features taken from different approaches. Perspiration- based approaches try to infer dynamic information from a single static impression. A combination of perspiration- and morphology- based features is then expected to achieve better performance than any of the individual measures improving the vitality detection accuracy.The proposed algorithm has been tested on three different optical sensors. Since our method does not require additional hardware, the cost of the fingerprint sensor does not increase.

- Lavanya (2011) [9]. In this paper, the fingerprint is segmented into four cells. The DCT is applied on each cell. The Harr Wavelet is applied on DCT coefficient of each cell. The directional information features and center area features are computed on LL sub band. The final Feature Vector is obtained by concatenating Directional Information and Centre Area Features. It is observed that the values of TSR and FRR are better in the case of proposed algorithm compared to existing algorithm.

- Rajkumar (2012) [10]. Present an effective algorithm for the fingerprint minutiae extraction with a secondary enhancement in the preprocessing stage, using a Gaussian filter after Fast Fourier transformation. The Gaussian filter makes the ridge smoothness and reduces the "hairy" structure in the ridge at the thinning process. This gives a less effort in the post processing stage. The total error rate of the proposed algorithm is within experimental limits and the proposed algorithm shows that the accuracy of fingerprint matching is reasonably improved.

- Ramachandra (2012) [11]. In this paper The variance of each block is determined by segmenting the finger print into $8 * 8$ blocks. Area of Interest (AOI) is obtained by removing the blocks with minimum variance. Features of Finger Print is obtained by applying Discrete Cosine Transform (dct) on AOI and converted to major and minor non-overlapping blocks to determine variance. The percentage recognition rate is better in the proposed algorithm compared to the existing algorithms.

- DCT coefficients are obtained for the region around a core point in which more inter-ridge variation is observed and are segmented into major and minor blocks.Please use a 9-point Times Roman font, or other Roman font with serifs, as close as possible in 
appearance to Times Roman in which these guidelines have been set. The goal is to have a 9-point text, as you see here. Please use sans-serif or non-proportional fonts only for special purposes, such as distinguishing source code text. If Times Roman is not available, try the font named Computer Modern Roman. On a Macintosh, use the font named Times. Right margins should be justified, not ragged.

\subsection{Fingerprint Representation}

Fingerprints are fully formed at about 7 months of fetus development. Fingerprint formation is similar to the growth of capillaries and blood vessels in angiogenesis. The general characteristics of the fingerprint emerge as the skin on the fingertip begins to differentiate. The finer details are determined by this changing microenvironment. There are so many variations during the formation of fingerprints that it would be virtually impossible for two fingerprints to be exactly alike. A fingerprint is a pattern of curving line structures called ridges, where the skin has a higher profile than its surroundings, which are called the valleys. The ridges are black and the valleys are white. Fingerprint representations rely on locations of the minutiae and the directions of ridges at the minutiae location. It includes information about locations of critical points (e.g., core and delta) [17]..

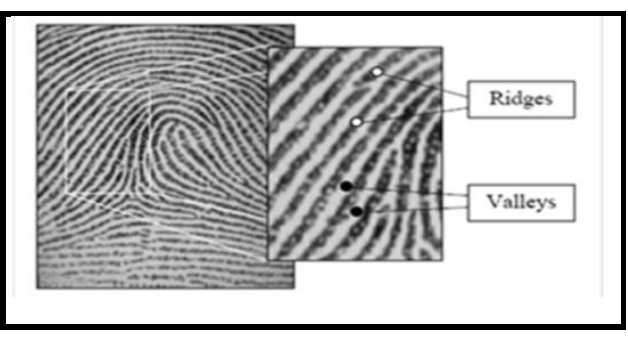

Fig (1): Fingerprint Representation

\section{FINGERPRINT CLASSIFICATION}

Fingerprint classification is a technical choice to reduce the number of comparisons during fingerprint retrieval and consequently to reduce the response time of the identification process [17]. The fingerprints have been traditionally classified into categories based on information in the global patterns of ridges [4].

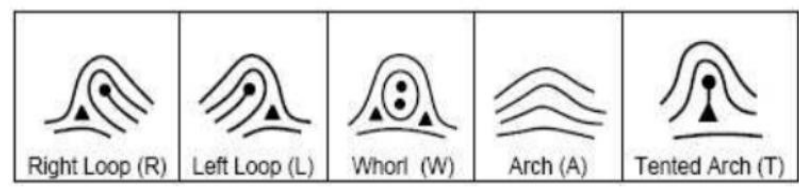

Fig (2): Patterns of fingerprint

\section{FINGERPRINT RECOGNITION ALGORITHM}

In this research the fingerprint image is passing into number of stages during the above phases. The work of implemented system is divided into two main phases. First is the training phase, and second the test phase.

Discrete Cosine Transform algorithm is implemented at the first five pictures belongs to each user. The result information generated by the algorithm is stored in the database to be further used by the second phase. The main object of this research is to create a fingerprint recognition system using the
MATLAB environment and using dct in feature extraction stage.
Training phase

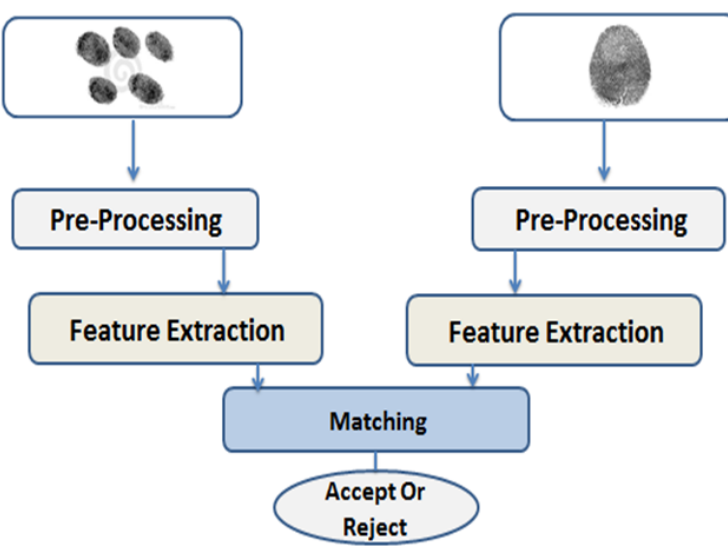

Figure (3): Diagram of the implemented fingerprint recognition system
- Database capture stages: The fingerprint database used in implemented system consists of pictures belong to 20 different users (10 males and 10 females). Fingerprint acquisitions by right and left thumbs (using ink).

- Preprocessing Stages: The performance of a fingerprint features extraction and matching algorithm depends heavily upon the quality of the input fingerprint image. to reduce the noise and increase the contrast between ridges and valleys. The fingerprint gray image is enhanced using median filter.

- $\quad$ Read an image

$\mathrm{S}=$ imread (path)

Image $=$ cropping $($ Image $)$

- Converting Color Image to Gray Scale Image

$\mathrm{IMG}=$ rgb2gray (image)

- $\quad$ Image Size

Image $=$ imresize (image, $[\mathrm{x}, \mathrm{y}])$;

Where $\mathrm{x}$ and $\mathrm{y}$ equals $\quad 256 * 256$

- Enhancement: The output of digital image enhancement is to a processed image that is used for a given application.

Median Filtering: The median is just the middle value of all the values of the pixels in the neighborhood.

$$
\begin{aligned}
& \mathrm{IMG}=\text { medfilt } 2(\text { image }) \\
& \text { Img }=\text { meanfilt } 2 \text { (image) and used } \mathrm{m}=\mathrm{std} 2 \text { (image) }
\end{aligned}
$$

- Feature Extraction Stage: Discrete cosine transforms (dct), are used for extracting features from the image data considering all the 2D-DCT coefficients to form the feature vectors for these two images, a measure of similarity can be obtained by using correlation.

DCT is a technique to convert data of the image into its elementary frequency components. DCT convert images from time (spatial) domain to (frequency) domain to decor relate pixels. (dct) helps separate the image into parts. The proposed 


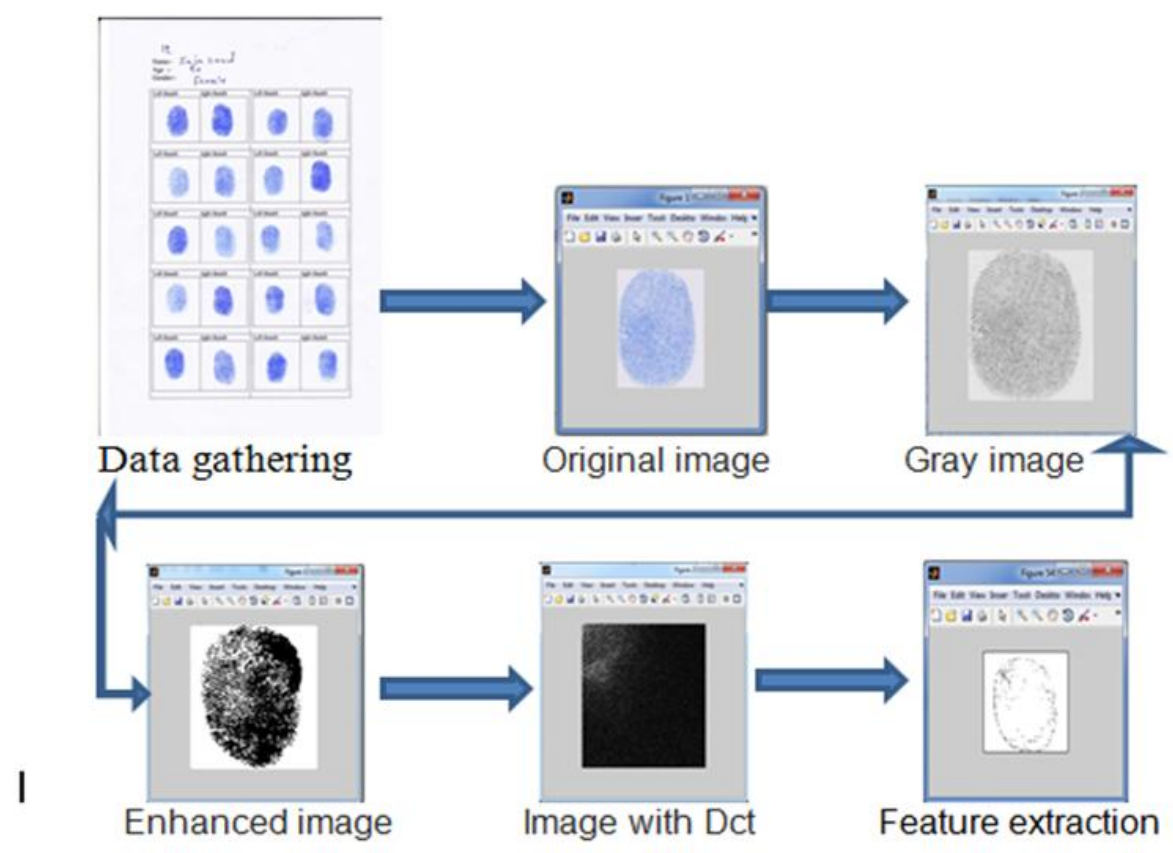

Figure (4): stage of Preprocessing and feature extraction

- Discrete Cosine Transform (DCT)

DCT convert images from time (spatial) domain to (frequency) domain to decor relate pixels [19]. (dct) helps separate the image into parts. The proposed scheme uses Discrete Cosine Transform (dct) to create feature vector for fingerprints. $\mathrm{N} 2$ pixels wide by $\mathrm{N} 1$ pixels high. A (i,j) is the intensity of the pixel in row $\mathrm{i}$ and column $\mathrm{j} ; \mathrm{B}(\mathrm{k} 1, \mathrm{k} 2)$ is the DCT coefficient in row $\mathrm{k} 1$ and column $\mathrm{k} 2$ of the dct. Each image is divided into sub blocks with $(\mathrm{N} \mathrm{x} \mathrm{N})$ size matrix.

$B\left(k_{1}, k_{2}\right)=\sum_{i=0}^{N_{1}-1} \sum_{j=0}^{N_{2}-1} 4 \cdot A(i, j) \cdot \cos \left[\frac{\pi \cdot k_{1}}{2 \cdot N_{1}} \cdot(2 \cdot i+1) \mid \cos \left[\frac{\pi \cdot k_{2}}{2 \cdot N_{2}} \cdot(2 \cdot j+1) \mid\right.\right.$

\section{- Matching stage}

A (three-class) categorization of fingerprint matching approaches:

a) Correlation-based matching: two fingerprint images are superimposed and the correlation between corresponding pixels is computed for different alignments (e.g., various displacements and rotations).

b) Minutiae-based matching: Minutiae matching essentially consist of finding the alignment between the template and the input minutiae sets that result in the maximum number of minutiae pairings.

c) Non-minutiae feature-based matching: minutiae extraction is difficult in extremely low-quality fingerprint images, whereas other features of the fingerprint ridge pattern [5].
In this study, the matching process was accomplished using correlation test, the results showed that the ratio of matching for fingerprint images of the same person would have been acceptable .

Table (1) result of matching first left fingerprint with five fingerprints

\begin{tabular}{|c|c|c|c|c|c|}
\hline & Corr & Corr & Corr & Corr & Corr \\
\hline Person 1 & $\mathbf{1}$ & $\mathbf{0 . 5 7 8 2}$ & $\mathbf{0 . 5 6 8 8}$ & $\mathbf{0 . 5 7 7 4}$ & $\mathbf{0 . 6 3 0 5}$ \\
\hline Person 2 & $\mathbf{1}$ & $\mathbf{0 . 5 5 4 7}$ & $\mathbf{0 . 5 7 7 5}$ & $\mathbf{0 . 6 2 9 6}$ & $\mathbf{0 . 7 2 9 6}$ \\
\hline Person 3 & $\mathbf{1}$ & $\mathbf{0 . 5 6 8 5}$ & $\mathbf{0 . 6 7 1 3}$ & $\mathbf{0 . 7 1 4 5}$ & $\mathbf{0 . 7 8 7 6}$ \\
\hline Person 4 & $\mathbf{1}$ & $\mathbf{0 . 6 7 7 4}$ & $\mathbf{0 . 6 7 1 4}$ & $\mathbf{0 . 7 7 6 8 9}$ & $\mathbf{0 . 7 9 6 3}$ \\
\hline Person 5 & 1 & $\mathbf{0 . 7 1 0 0}$ & $\mathbf{0 . 7 2 6 4}$ & $\mathbf{0 . 7 9 9 8}$ & $\mathbf{0 . 8 4 3 3}$ \\
\hline
\end{tabular}

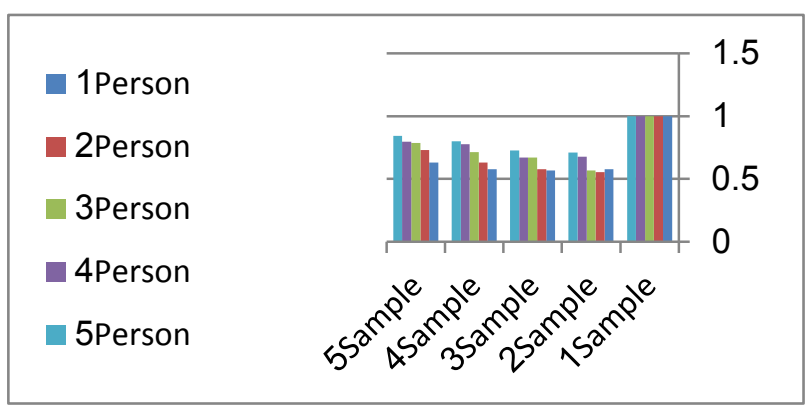

Figure (5) illustrate the result of matching. 


\section{CONCLUSIONS}

Fingerprint identification is one of the most well-known and publicized biometrics. Because of their uniqueness and consistency over time, fingerprints have been used for identification for over a century. In this study, DCT is applied to the entire image. This gives DCT coefficients, which are global features fingerprint recognition approach based on discrete cosine transform technique, is proposed. This algorithm based on the matching process via correlation in the images, and then utilizes DCT technique to extract important features. This study faces many problems like low quality of fingerprint input image

\section{REFERENCES}

[1] Muzhir Sh. Al-Ani, Isra H. Al-Ani," Gait Recognition Based Improved Histogram", Journal of Emerging Trends in Computing and Information Sciences, VOL. 2, NO. 12, December 2011.

[2] Marios Savvides Carnegie Mellon CyLab \& ECE, "Introduction to Biometric Recognition Technologies and Applications ".

[3] Jays Close, Viables "An introduction to biometrics", Motorola, Limited. Industrial Estate Basingstoke Hampshire, Rg 22, 4PG UNITED KINGDOM, August 2006.

[4] Martin Hanneghan, Biometrics and security, CMPCD1030 Computing and Society,11-2010, Liverpool John Moores University.

[5] Maltoni, D. Maio, A. K. Jain, and S. Prabhakar (Eds.), "Handbook of Fingerprint Recognition ", 2009,second edition, Springer-Verlag.

[6] Helal. Mahmoud, "Fingerprint recognition using fractal geometry", MSc thesis, Al-Anbar University, February 2011.

[7] Abdullah .C, avus_og־lu, Salih Go“rgünog־lu, "A fast fingerprint image enhancement algorithm using a parabolic mask ", Computers and Electrical Engineering ,34 (2008)

[8] Emanuela Marasco a,b, Carlo Sansone Pattern Recognition Letters,"Combining perspiration- and morphology-based static features for fingerprint livens detection", 33 (2012) 1148-1156.

[9] Lavanya B N1 and K B Raja1, "Performance Evaluation of Fingerprint Identification Based on DCT and DWT using Multiple Matching Techniques". IJCSI International Journal of Computer Science Issues, Vol. 8, No 1, 2011.

[10] Raju Rajkumar1, K Hemachandran2, "A Secondary Fingerprint Enhancement and Minutiae Extraction", Department of Computer Science, Assam University, Silchar, India, 2009.

[11] Ramachandra A C, K B Raja, Venugopal K R, L M Patnaik, " Non Minitia Fingerprint Recognition based on Segmentation ", International Journal of IssueInnovative Technology and Exploring Engineering (IJITEE), Vol. 1, July 2012.

[12] Indra Devi," Efficient Fingerprint Recognition Through Improvement of Feature Level Clustering ", Indexing and Matching Using Discrete Cosine Transform, Indra
Ganesan College of Engineering, Tiruchirappalli ,India 2010.

[13] Asker M. Bazen, "Fingerprint Identification - Feature Extraction, Matching, and Database Search", August 19, 2002.

[14] Kulwinder Singh, Kiranbir Kaur, Ashok Sardana , "Fingerprint Feature Extraction ", Gulzar Group of Institutes, Khanna, Punjab, India 2010.

[15] T. Liu, G. Zhu, C. Zhang and P. Hao, "Fingerprint indexing based on Singular points ", International Conference on Image Processing 3, Septembe 2005.

[16] Allam Mousa \& Zain S. " Barham Fingerprint Recognition using MATLAB", Graduation project Prepared, d voice characteristics.

[17] Fayadh, Adnan Maroof, Abed "Fingerprint image Prepost processing Methods for minutiae extraction ". College of Computer and mathematics science. Tikrit University.

[18] Lin Hong, Student Member, IEEE, Yifei Wan, and Anil Jain, Fellow, IEEE," Fingerprint Image Enhancement", IEEE transaction on pattern analysis and machine intelligence, vol. 20, no. 8, august, 1998.

[19] Hafiz Imtiaz, Shubhra Aich, Shaikh Anowarul Fattah," A Novel Pre-processing Technique for DCT-domain Palmprint Recognition", International Journal of Scientific \& Technology Research Volume 1, Issue 3, 2012.

[20] Kim-chyan Gan," Discrete Cosine Transform Basics ", Freescale Semiconductor Application Note, 11/2004

\section{AUTHORS PROFILE}

${ }^{1}$ Muzhir Shaban Al-Ani has received Ph.D. in Computer \&Communication Engineering Technology, ETSII, Valladolid, Spain, 1994. Assistant of Dean at AlAnbar Technical Institute (1985). Head of Electrical Department at Al-Anbar Technical Institute, Iraq (19851988), Head of Computer and Software Engineering Department at Al-Mustansyria University, Iraq (19972001), Dean of Computer Science (CS) \& Information System (IS) faculty at University of Technology, Iraq (2001-2003). He joined in 15 September 2003 Electrical and Computer Engineering Department, College of Engineering, Applied Science University, Amman, Jordan, as Associated Professor. He joined in 15 September 2005 Management Information System Department, Amman Arab University, Amman, Jordan, as Associated Professor, then he joined computer science department in 15 September 2008 at the same university

${ }^{2}$ Wasan Maddah Al- Aloosi has received B.Sc. in administration \& economic, Baghdad university (19901991), B.Sc. in Computer Science in Al-Maaref University College (2009-2010), Anbar, Iraq. M.Sc student (2012- tell now) in Computer Science Department, Al-Anbar University. 